\title{
Measurement of light-ion production at the new Uppsala neutron beam facility
} \author{
V. Simutkin ${ }^{2}$, P.A. Söderström ${ }^{2}$, and U. Tippawan ${ }^{4}$ \\ ${ }^{1}$ Department of Advanced Energy Engineering, Kyushu University, Kasuga, Fukuoka 816-8580, Japan \\ 2 Department of Neutron Research, Uppsala University, P.O. Box 525, 75120 Uppsala, Sweden \\ 3 The Svedberg Laboratory, Uppsala University, P.O. Box 533, 75121 Uppsala, Sweden \\ ${ }^{4}$ Fast Neutron Research Facility, Chiang Mai University, P.O. Box 217, Chiang Mai 500200, Thailand
}

M. Hayashi ${ }^{1, \mathrm{a}}$, Y. Watanabe ${ }^{1}$, R. Bevilacqua ${ }^{2}$, J. Blomgren $^{2}$, L. Nilsson ${ }^{2}$, A. Öhrn ${ }^{2}$, M. Österlund ${ }^{2}$, S. Pomp $^{2}$, A. Prokofiev $^{3}$,

\begin{abstract}
A collaborative research project has been launched on neutron-induced light-ion production measurements using the new Uppsala neutron beam facility. The available energy range of quasi mono-energetic neutron beams is extended up to $175 \mathrm{MeV}$. Double-differential cross sections (DDXs) of light-ion production (p, d, t, ${ }^{3} \mathrm{He}$, and $\alpha$ ) are measured using a conventional spectrometer system which consists of eight counter telescopes. Each telescope is composed of two silicon surface barrier detectors as the $\Delta \mathrm{E}$ detectors and a $\mathrm{CsI}(\mathrm{Tl})$ scintillator as the $\mathrm{E}$ detector. Response of the scintillators to $160 \mathrm{MeV}$ protons is measured to test the performance. The measured response is reproduced well by a PHITS transport calculation. The DDXs of light-ion production are measured for $\mathrm{Ca}$ at $94 \mathrm{MeV}$ and $\mathrm{C}$ at $175 \mathrm{MeV}$ at angles between $20^{\circ}$ to $160^{\circ}$ in steps of $20^{\circ}$. The preliminary experimental (n,xp) data are shown in comparison with a model calculation using the TALYS code and the evaluated cross sections in the JENDL high-energy file.
\end{abstract}

\section{Introduction}

Recently, there have been increasing nuclear data needs for neutron-induced light-ion production at intermediate energies, especially 20 to $200 \mathrm{MeV}$, for a wide variety of applications, such as radiation treatment of cancer therapy, neutron dosimetry at high altitude and space, single event effects in microelectronics, and accelerator-driven transmutation of nuclear waste. To satisfy these needs, a series of experiments have successfully been performed for several targets $(\mathrm{C}, \mathrm{O}$, $\mathrm{Si}, \mathrm{Fe}, \mathrm{Pb}$, and $\mathrm{U}$ ) at $96 \mathrm{MeV}$ using the quasi mono-energetic neutron facility at the The Svedberg Laboratory (TSL) [1-3]. Similar light-ion production measurements have been carried in the incident energy range below $100 \mathrm{MeV}$ at other facilities as well $[4,5]$. However, there has been no systematic measurement at energies between 100 and $200 \mathrm{MeV}$ until now.

The Uppsala neutron beam facility has recently been upgraded [6], so that quasi mono-energetic neutron beams are available with higher intensity than the previous one for energies up to $175 \mathrm{MeV}$. Using the new facility, systematic measurements have been planned of double-differential cross sections (DDXs) for light-ion production induced by $175 \mathrm{MeV}$ neutrons. The MEDLEY spectrometer setup used in the previous experiments at $96 \mathrm{MeV}[1,3]$ is partly modified by installing such a thick CsI scintillator as the E-detector that light ions generated from reactions by $175 \mathrm{MeV}$ neutrons are fully stopped in the scintillator. In the present work, some preliminary measurements are performed as the feasibility demonstration. One of them is to measure the response of CsI scintillators to $160 \mathrm{MeV}$ protons as the performance evaluation test, i.e., estimation of the reaction tail. The DDXs of $(n, x p)$ reactions on $\mathrm{Ca}$ at $94 \mathrm{MeV}$ (hereafter, measurement-I) and on $\mathrm{C}$ at $175 \mathrm{MeV}$ (measurement-II) are measured using the

\footnotetext{
${ }^{a}$ Corresponding author, e-mail: teru@aees. kyushu-u.ac .jp
}

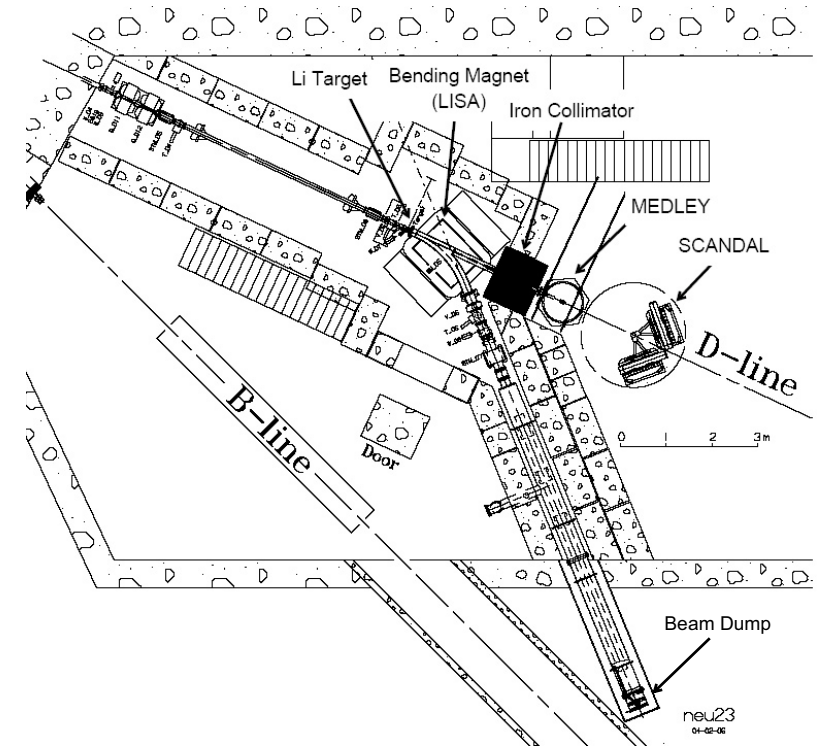

Fig. 1. New neutron beam facility at TSL and experimental setup.

MEDLEY setup. The experimental DDXs for $\mathrm{Ca}$ are compared with a model calculation using the TALYS code [7]. Also, a preliminary result is presented for comparison between the measured $\mathrm{C}(\mathrm{n}, \mathrm{xp})$ spectrum at $20^{\circ}$ and the evaluated cross sections in the JENDL high-energy file [8].

\section{Experimental methods}

Figure 1 shows the new Uppsala neutron beam facility [6] at the TSL and the experimental setup of MEDLEY experiments [9]. Protons from the cyclotron impinge on an enriched ${ }^{7} \mathrm{Li}$ target, and neutrons are produced by the ${ }^{7} \mathrm{Li}(\mathrm{p}, \mathrm{n}){ }^{7} \mathrm{Be}$ 
reaction. In measurement-I, the $98.5 \mathrm{MeV}$ protons produces neutrons with a peak energy of $94 \mathrm{MeV}$ using a lithium target $8 \mathrm{~mm}$ thick, while the $180 \mathrm{MeV}$ protons produces neutrons with a peak energy of $175 \mathrm{MeV}$ using a $23.5 \mathrm{~mm}$ thick target in measurement-II. The neutrons are transported to the MEDLEY chamber passing through a 100-cm long and conical iron collimator whose diameter is $54 \mathrm{~mm}$ at the end of collimator. The neutron flux was about $3.1 \times 10^{5} \mathrm{n} /\left(\mathrm{cm}^{2} \mathrm{~s}\right)$ with a proton beam intensity of $3.4 \mu \mathrm{A}$ in measurement-I, while it was about $4.3 \times 10^{4} \mathrm{n} /\left(\mathrm{cm}^{2} \mathrm{~s}\right)$ with a proton beam intensity of $0.3 \mu \mathrm{A}$ in measurement-II. The proton beam passing through the Li target is deflected by a bending magnet into the beam dump and integrated in a Faraday cap in order to monitor the beam current. In addition, a pre-collimator made out of lead blocks was installed temporarily inside the bending magnet to reduce a background component and improve the signal to background ratio. The distance from the $\mathrm{Li}$ target to the center of MEDLEY chamber was $3.74 \mathrm{~m}$. The relative neutron beam intensity is monitored by the integrated proton beam current at the beam dump and by both a thin film breakdown counter and an ionization chamber mounted downstream of the MEDLEY setup.

The MEDLEY setup and construction details of each telescope are illustrated in figure 2. MEDLEY consists of eight three-element telescopes mounted inside a vacuum chamber with a diameter of $90 \mathrm{~cm}$. Each telescope consists of two fully depleted silicon surface barrier detectors serving as $\Delta \mathrm{E}$ detector and a $\mathrm{CsI}(\mathrm{Tl})$ scintillator serving as $\mathrm{E}$ detector. The thickness of the $\Delta \mathrm{E}$ detectors is in the range of $50-60 \mu \mathrm{m}$ for the first one, and $400-500 \mu \mathrm{m}$ for the second one. The $\mathrm{CsI}(\mathrm{Tl})$ scintillators were upgraded from the previous ones [13], and have a total length of $100 \mathrm{~mm}$ which is enough to stop high-energy protons produced in the $175 \mathrm{MeV}$ measurement. They have a cylindrical shape with $50 \mathrm{~mm}$ diameter, where the last $30 \mathrm{~mm}$ are tapered to $18 \mathrm{~mm}$ diameter to match the size of a Hamamatsu S3204-08 photodiode for the light readout. The signals from each telescope are processed using the same data acquisition system as in the previous $96-\mathrm{MeV}$ measurements [1-3].

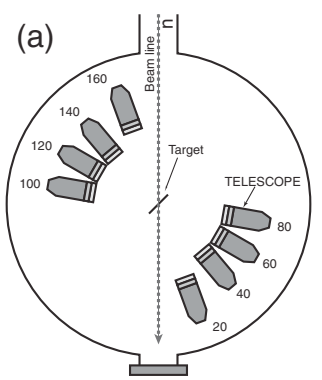

(b)

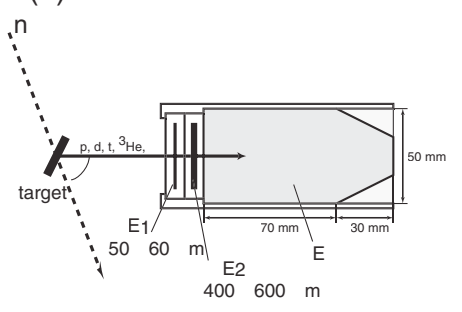

Fig. 2. MEDLEY setup: (a) arrangement of eight telescopes inside the MEDLEY chamber, and (b) construction details of each telescope.

Calcium and carbon targets are placed at the center of the MEDLEY chamber. The carbon target was $22 \mathrm{~mm}$ in diameter and $1.0 \mathrm{~mm}$ thick, and the calcium target was $29 \mathrm{~mm}$ in diameter and $230 \mu \mathrm{m}$ thick. For absolute cross section normalization, a polyethylene $\left(\mathrm{CH}_{2}\right)$ target with $25 \mathrm{~mm}$ diameter and $1.0 \mathrm{~mm}$ thickness was used. Instrumental backgrounds are also measured by removing the target from the neutron beam.

Since the ${ }^{7} \mathrm{Li}(\mathrm{p}, \mathrm{n})$ reaction produces peak neutrons and low-energy tail neutrons, time-of-flight (TOF) measurements are used to reject the tail neutrons. The TOF data are measured as a time difference between master trigger signal and RF timing signal from the cyclotron.

\section{Data reduction procedure}

The data reduction procedure based on the $\Delta \mathrm{E}-\mathrm{E}$ technique is the same as in the previous $96-\mathrm{MeV}$ measurement and explained in detail in refs. $[1,3,16]$. Here the procedure for the measurement-II is briefly described.

Energy calibration of all detectors is obtained using the data themselves. Events in the $\triangle \mathrm{E}-\mathrm{E}$ bands are fitted with respect to the energy deposited in the two $\Delta \mathrm{E}$ silicon detectors, which is determined from the thicknesses and the energy losses calculated with the SRIM code [10]. One example is shown in figure 3(a) for the present $175-\mathrm{MeV}$ measurement.
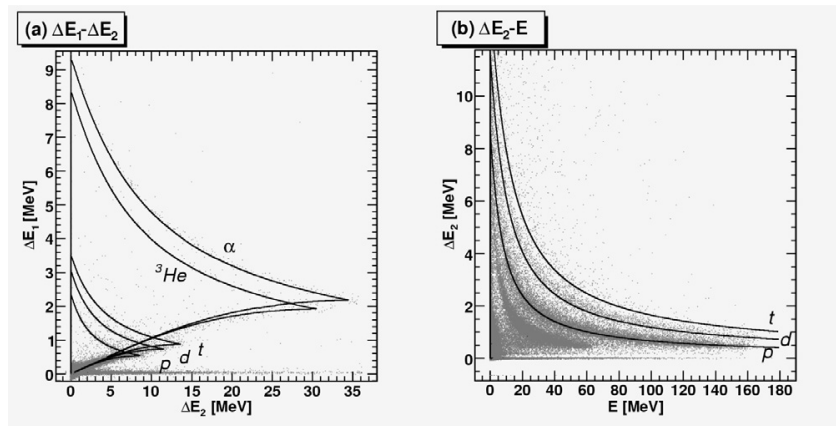

Fig. 3. Calibrated $\Delta E_{1}-\Delta E_{2}$ plot for telescope 1 at 20 degree in the left panel (a). The solid lines correspond to calculation results of energy-loss values for proton, deuteron, triton, ${ }^{3} \mathrm{He}$ and $\alpha$ particles. Calibrated $\Delta E_{2}-E$ plot for telescope 1 at 20 degree in the right panel (b). The solid line corresponds to calculated energy-loss values for protons.

For the energy calibration of the $\mathrm{CsI}(\mathrm{Tl})$ scintillator, the following approximate expression is applied to hydrogen isotopes [9]:

$$
E=a+b L+c(b L)^{2},
$$

where $L$ is the light output and $a, b$, and $c$ are the fitting parameters. The parameter $c$ depends on the kind of charged particles. For the small CsI(Tl) scintillators, the $c$ parameter was found by Tippawan [12] to be 0.0032 for protons. For the new $\mathrm{CsI}(\mathrm{Tl})$ scintillators, the derived $c$ parameter was found to be $\sim 0.001$ for protons. Figure 3(b) shows a result of the energy calibration for $\Delta E_{2}$ and $E$ detectors for the $175-\mathrm{MeV}$ measurement.

The measured TOF data are used for selection of lightion events induced by neutrons in the main peak of the source neutron spectrum.

Some corrections are necessary to obtain final DDX data. Background events measured in target-out runs are subtracted from the target-in runs after normalization to the same neutron 
fluence. Corrections due to the finite target thickness are made in the method described in ref. [1]. However, it should be noted that this correction has not yet been applied in the present analysis of the $175-\mathrm{MeV}$ measurement. The finite efficiency of the $\mathrm{CsI}(\mathrm{Tl})$ scintillator is corrected using the Monte Carlo simulation method discussed below.

The number of the net counts due to $\mathrm{np}$ scattering is obtained using measurements of the (n,p) spectrum at $20^{\circ}$ for both the targets, $\mathrm{CH}_{2}$ and $\mathrm{C}$. The result is shown in figure 4. Finally, the absolute values of the measured cross sections are determined using the reference $\mathrm{np}$ cross sections in the same method as in refs. $[1,3]$. The np scattering cross sections are taken from NN-online [13].
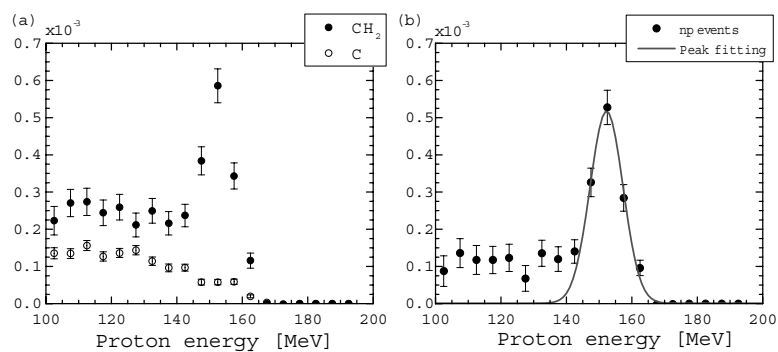

Fig. 4. Measured np scattering peak: (a) the contribution from $\mathrm{C}$ in the $\mathrm{CH}_{2}$ data and (b) the net np peak with a Gaussian fitting.

\section{Results and discussion}

\subsection{Response of CsI(TI) scintillator to protons}

The performance of the newly-installed CsI(Tl) scintillators was investigated using a $160-\mathrm{MeV}$ proton beam with very low intensity, i.e., about 100 protons per second, prior to the $175 \mathrm{MeV}$ neutron measurement. The proton beam was formed by installing a collimator system into the B-line shown in figure 1, which consists of a combination of a tantalum scatterer with a thickness of $4 \mathrm{~mm}$ and a graphite collimator with a diameter of $4 \mathrm{~mm}$. The counter telescope depicted in figure 2(b) was placed near the exit of the B-line to measure the response of the $\mathrm{Cs}(\mathrm{Tl})$ scintillator to $160-\mathrm{MeV}$ protons.

In figure 5(a), the measured proton spectrum normalized to the observed peak corresponding to $160 \mathrm{MeV}$ is presented together with a Monte Carlo simulation using the PHITS code [14]. Note that the energy resolution of the oberved peak was $1.7 \mathrm{MeV}$ in FWHM, although it is not shown in the figure. Due to nuclear interactions a certain fraction of incident protons does not fully deposit their energies into the $\mathrm{CsI}(\mathrm{Tl})$ scintillator. This leads to the so-called reaction tail which is seen in the low-energy region. The PHITS simulation reproduces the measurement well. The fraction of the reaction tail for simulated and experimental data is plotted as a function of proton energy in figure 5(b). The PHITS simulation is in excellent agreement with both the previous work [15] given by the solid line and the present measurement denoted by the full circle with the error. The efficiency of the $\mathrm{CsI}(\mathrm{Tl})$ is finally corrected using the PHITS calculation in the data analysis of the $\mathrm{C}(\mathrm{n}, \mathrm{xp})$ reaction at $175 \mathrm{MeV}$.
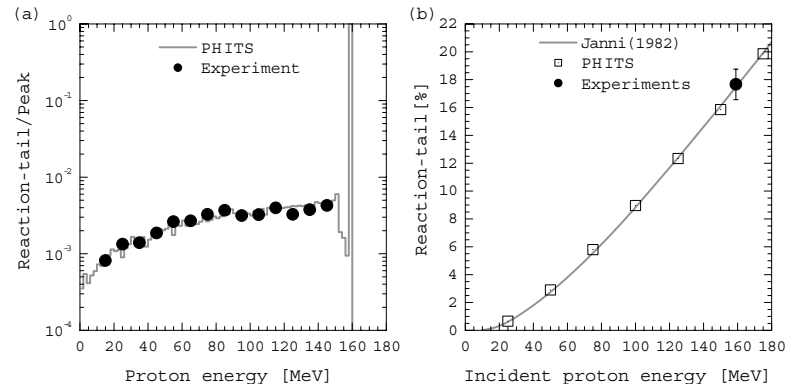

Fig. 5. Measured response of $\mathrm{CsI}(\mathrm{Tl})$ scintillator to $160 \mathrm{MeV}$ protons: (a) the ratio of reaction tail to peak with the PHITS calculation, and (b) the fraction of the reaction tail as a function of incident proton energy. The explanation of the symbols and lines is given in the text.

\section{2 $\mathrm{Ca}(\mathrm{n}, \mathrm{xp})$ at $94 \mathrm{MeV}$}

In figure 6, experimental double-differential cross sections of the $94 \mathrm{MeV}(\mathrm{n}, \mathrm{xp})$ reaction on $\mathrm{Ca}$ are presented for four angles and compared with a model calculation using the TALYS0.64 code [7]. The detail of the data analysis and the model calculation is reported elsewhere [16]. The TALYS calculation overestimates the low energy region where the evaporation process is dominant, while underestimating the intermediate continuum region at forward angles where the preequilibrium emission is expected to have a large contribution.

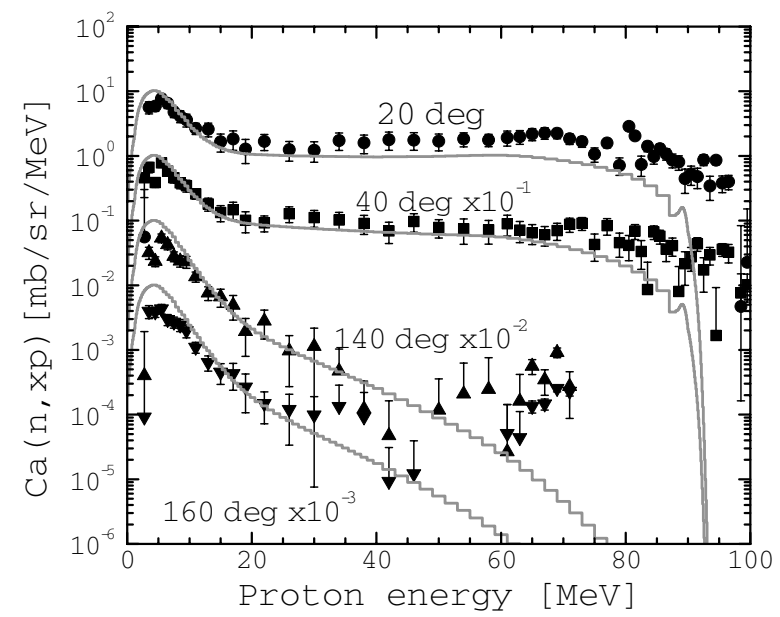

Fig. 6. Comparison of experimental double-differential cross sections for the $\mathrm{Ca}(\mathrm{n}, \mathrm{xp})$ reaction at $94 \mathrm{MeV}$ (filled symbols with error bar) with the TALYS-0.64 calculation (solid histograms).

\section{3 $\mathrm{C}(\mathrm{n}, \mathrm{xp})$ at $175 \mathrm{MeV}$}

The incident neutron spectrum accepted by the TOF gate was estimated from data analysis of the recoil protons from $\mathrm{np}$ scattering in the measurement of the $\mathrm{CH}_{2}$ target. Figure 7 shows the result together with the source neutron spectrum calculated using an empirical formula [17]. Both the spectra are normalized so that each peak corresponding to $175 \mathrm{MeV}$ is unity. The calculated spectrum is folded using a Gaussian function with the same width as the experimental energy resolution. The measured neutron spectrum is in good agreement 
with the calculated one. The hatched region above $95 \mathrm{MeV}$ corresponds to the accepted neutron spectrum in the present measurement.

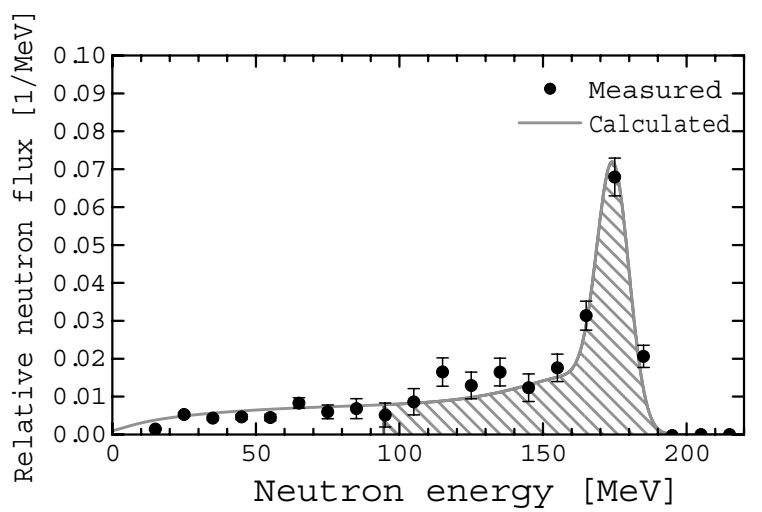

Fig. 7. Comparison between the measured neutron spectrum and calculated one. The hatched region corresponds to the accepted neutron spectrum.

The $\mathrm{C}(\mathrm{n}, \mathrm{xp})$ spectrum measured at $20^{\circ}$ is shown in figure 8 . The measured spectrum is compared with a folding spectrum $\sigma^{\text {cal }}\left(E_{p}, \theta_{p}\right)$ obtained by the following equation:

$$
\sigma^{c a l}\left(E_{p}, \theta_{p}\right)=\int_{95 M e V}^{175 M e V} \sigma^{\text {eval }}\left(E_{n}, E_{p}, \theta_{p}\right) f\left(E_{n}\right) d E_{n}
$$

where $f\left(E_{n}\right)$ is the accepted source neutron spectrum shown in figure 7 and the calculated one [17] is used. The JENDL/HE evaluated cross sections [8] are used as $\sigma^{\text {eval }}\left(E_{n}, E_{p}, \theta_{p}\right)$ in equation (2). As can be seen in figure 8 , the calculated $(n, x p)$ spectrum shows good agreement with the measured one in the intermediate proton energy range.

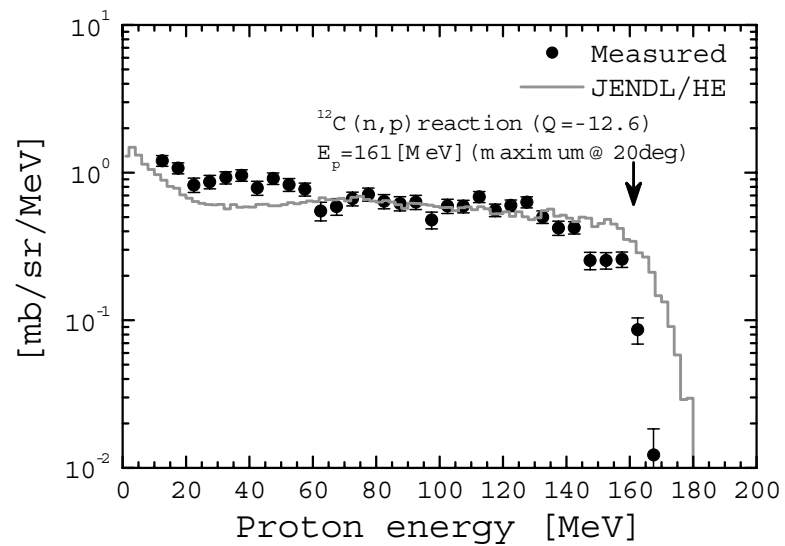

Fig. 8. Comparison of the experimental $\mathrm{C}(\mathrm{n}, \mathrm{xp})$ spectrum at $20^{\circ}$ with the folding spectrum calculated from the JENDL/HE evaluated cross sections and the accepcted neutron spectrum.

\section{Summary}

We have measured the double-differential cross sections of $(\mathrm{n}, \mathrm{xp})$ reactions on $\mathrm{Ca}$ at $94 \mathrm{MeV}$ and on $\mathrm{C}$ at $175 \mathrm{MeV}$ using the new Uppsala neutron beam facility for the first time. The measured $\mathrm{Ca}$ data were in reasonable agreement with the TALYS calculation, although the calculation overestimated the evaporation region at all angles and underestimated the preequilibrium region at $20^{\circ}$. Since the background contribution from source neutrons having continuous energies between $95 \mathrm{MeV}$ and $175 \mathrm{MeV}$ could not be subtracted from the measured proton spectra for $\mathrm{C}$, the present measurement was compared with the proton spectrum obtained by folding the JENDL high-energy data and the expected source neutron spectrum. The proton spectrum measured at $20^{\circ}$ showed overall good agreement with the JENDL high-energy data. Further analysis including other angles will be required for detailed comparison. In addition, it was confirmed that the measured response of the new $\mathrm{CsI}(\mathrm{Tl})$ scintillators to $160 \mathrm{MeV}$ proton was reproduced well by the PHITS transport calculation.

A series of light-ion production measurements is planned for other targets, $\mathrm{O}, \mathrm{Si}, \mathrm{Fe}, \mathrm{Pb}$, and $\mathrm{U}$, at $175 \mathrm{MeV}$ in order to meet nuclear data needs for fast neutron applications.

M.H. is grateful to the Kyushu Industrial Technology Center for the Grants-in-Aid for human resource development.

\section{References}

1. U. Tippawan et al., Phys. Rev. C 69, 064609 (2004).

2. V. Blideanu et al., Phys. Rev. C 70, 014607 (2004).

3. U. Tippawan et al., Phys. Rev. C 73, 034611 (2006).

4. Y. Nauchi et al., J. Nucl. Sci. Technol. 36, 143 (1999).

5. I. Slypen et al., Phys. Rev. C 51, 1303 (1995).

6. S. Pomp et al., in Proceedings of the International Conference on Nuclear Data for Science and Technology, Santa Fe, USA, Sept. 26-Oct. 1, edited by R.C. Haight, M.B. Chadwick, T. Kawano, P. Talou (AIP, New York, 2005), p. 780.

7. A.J. Koning, S. Hilaire, M.C. Duijvestijn, ibid., p. 1154.

8. Y. Watanabe et al., ibid., p. 326.

9. S. Dangtip et al., Nucl. Instrum. Meth. A 452, 484 (2000).

10. J.F. Ziegler et al., The Stopping and Range of Ion in Solids (Pergamon Press, New York, 1985).

11. D. Horn et al., Nucl. Instrum. Meth. A 320, 237 (1992).

12. U. Tippawan, Ph.D. thesis, Graduate School Chiang Mai University, Chiang Mai, May 2004, Thailand.

13. SAID Nucleon Nucleon scattering database. Available from Center for Nuclear Studies, Department of Physics, George Washington University, USA, URL: http://gwdac.phys.gwu.edu.

14. H. Iwase et al., J. Nucl. Sci. Technol. 39, 1142 (2002).

15. J.F. Janni, At. Data Nucl. Data Tables 27, 147 (1982).

16. P.A. Söderström, Diploma thesis, Uppsala University Neutron Physics Report, Feb. 2007.

17. A. Prokofiev et al., in Proceedings of the International Conference on Nuclear Data for Science and Technology, Tsukuba, Japan, Oct. 7-12, 2001, J. Nucl. Sci. Technol., Suppl. 2 (2002), p. 112. 